Metadata for the data produced and analysed for the article:

\title{
Particle balance and return loops for microplastics in a tertiary-level wastewater treatment plant
}

Pauliina Salmi $^{1 *}$, Kalle Ryymin ${ }^{2}$, Anna K. Karjalainen ${ }^{2}$, Anna Mikola ${ }^{3}$, Emilia Uurasjärvi ${ }^{4}$, Julia Talvitie ${ }^{5}$

\author{
${ }^{1}$ Faculty of Information Technology, Mattilanniemi 2 (Agora building), Po Box 35, FI-40014 University of Jyväskylä, Jyväskylä, \\ Finland, pauliina.u.m.salmi@jyu.fi *Corresponding author \\ 2Department of Biological and Environmental Science, Survontie 9C (YAC Building), Po Box 35, FI-40014, University of Jyväskylä, \\ kalle.ryymin@gmail.com \\ ${ }^{2}$ Department of Biological and Environmental Science, Survontie 9C (YAC Building), Po Box 35, FI-40014, University of Jyväskylä, \\ anna.k.karjalainen@jyu.fi \\ 3Department of Built Environment, Tietotie 1E, FI-00076 Aalto University, Espoo, Finland, anna.mikola@aalto.fi \\ ${ }^{4}$ SIB Labs Infrastructure Unit, University of Eastern Finland, P.O. Box 1627, 70211 Kuopio, Finland, emilia.uurasjarvi@uef.fi \\ ${ }^{5}$ Marine Management, Finnish Environment Institute (SYKE), Latokartanonkaari 11, FI-00790 Helsinki, Finland, \\ julia.talvitie@ymparisto.fi
}

The data processed and analysed in this study is in a file named Data_Nenainniemi.xlsx. All the data is compiled on the first sheet (Sheet1). For detailed description of the motives, methods, and results of this study, please see the published article. If you wish to use the data, please contact the corresponding author, or some of the other authors.

Column A shows the numeric ID of each sampling point in the Nenäinniemi WWTP and has values between 1-12. The numbers and sampling point names correspond those in the article's Figure 1 and Tables 2-3.

Column B shows the sample replicates and column $\mathrm{C}$ shows the sampling point names that correspond those in article's Table 1.

Columns D-F display the number of microplastics particles counted with Raman microscope as described in the Materials and Methods section of the article.

Column $\mathrm{G}$ shows sample volumes. Column $\mathrm{H}$ shows the proportion of the sample volume (in column $\mathrm{G}$ ) examined with the Raman microscopy.

Column I is the microplastics concentration calculated based on the given numbers of particles and sample volumes.

Column J values are means of examined sample replicates and $\mathrm{K}$ standard deviations.

Values in column $L$ refer to measured flow rate $(Q)$ as $\mathrm{m}^{3}$ per day in the Nenäinniemi WWTP.

Column $\mathrm{N}$ is daily load of microplastics from the given subprocess to a following one as number of microplastics $\times 10^{9}$ per day calculated using the mean concentrations and the $Q$-values given in the previous columns. Column $\mathrm{O}$ values are corresponding standard deviations. Column $\mathrm{P}$ is the daily load calculated for each replicate samples.

Column Q show the proportion of microplastics daily loads of the load to the Nenäinniemi WWTP via plant influent.

Column R shows whether Thermo Raman microscope DRX or DRX2i was used to examine the current sample. Red ' $x$ ' refers to the DXR model. 
Column S...AP show the material distribution for counted microplastics divided to fibre-like and fragmentlike particles.

Columns AQ...BN show the daily loads of each plastic material calculated using the sample volumes and proportions given in columns $\mathrm{G}$ and $\mathrm{H}$, flow rates given in column $\mathrm{L}$ and number of particles given in columns S...AP.

In columns BO...CP, data is arranged as mean daily loads of observed material on each sampling point to produce Figure 5 in the article.

Columns CQ...DD are data arrangements to produce Figure 5 and the bar charts embedded in Figures 2-3 in the article. The bar charts and Figure 5 are included in this Excel file. 